Article

\title{
A community engaged dental curriculum: a rural Indigenous outplacement programme
}

\author{
Menaka A. Abuzar, Julie Owen \\ Melbourne Dental School, University of Melbourne, Australia
}

Significance for public health

An oral health outreach programme is one of the suggested approaches to effectively address the endemic issues of poor oral health among Indigenous people around the world. An Indigenous dental clinical outplacement in Australia provides an example of beneficial outcomes of such an approach. It provides dental students with an opportunity to experience the health issues related to Australian Indigenous communities and prepare future graduates to work comfortably in the public health care system. Indigenous people also develop trust and feel comfortable in receiving oral health care services from non-Indigenous clinicians.

\section{Abstract}

Background. Indigenous people worldwide suffer from poor oral health as compared to non-Indigenous citizens. One of the approaches to bring about improvement in Indigenous oral health is to enhance the service provision by implementing oral health outplacement programmes. A case study of such a programme for dental students in Australia reports how an educational institution can successfully engage with an Indigenous oral health service to provide learning experiences to the students as well as deliver much needed services to the community.

Design and Methods. The assessment of this ongoing outplacement programme over the period of 2008-14, based on students' feedback, highlights some of the key beneficial outcomes. Students agreed that the Indigenous outplacement programme improved their understanding of Indigenous issues (mean \pm SD: $4.10 \pm 0.8$; 5 refers to strongly agree on 5-point scale) and increased the possibility that they will practise in Indigenous health $(3.66 \pm 1.0)$. They were pleased with the assistance received by clinical supervisors and clinic staff at the Indigenous dental clinic $(4.28 \pm 0.8)$.

Conclusions. This programme has demonstrated that structured student outplacements are valuable in building relations across cultures especially with Indigenous communities. It has also shown that university engagement with the public health sector can be beneficial to both institutions.

\section{Introduction}

Indigenous people around the world have relatively low standards of oral and general health. ${ }^{1}$ Oral health inequalities between Indigenous and non-Indigenous communities in the world are markedly similar irrespective of different geographical, cultural and historical backgrounds. ${ }^{2}$ Indigenous communities face considerable barriers to accessing oral health care since they live in rural and remote areas of their respective countries. They are further challenged by the shortage of culturally appropriate and family focussed care. The broad approaches adopted internationally to address these issues include dental clinical outplacements (outreach), integrated dental and primary health care services, understanding of traditional practices by health care professionals, and focussed service delivery including a team approach by oral health care professionals. ${ }^{3}$ This paper describes one of these approaches, i.e., Indigenous outplacement programme for dental students.

According to the World Health Organisation (WHO), Indigenous people are those who identify themselves as distinct minority groups, have historical continuity with pre-colonial societies, follow distinct socioeconomic systems, and maintain distinct languages and cultures. ${ }^{4}$ Many regions use different terms such as aboriginals, tribes and first people, which are synonymous to indigenous. ${ }^{5}$ In Australia, the Indigenous people include Aboriginals and Torres Strait Islanders. They constitute approximately $3 \%$ of the nation's population with a significant proportion of these individuals (43\%) residing in regional and remote areas. ${ }^{6}$

As in other parts of the world, Indigenous Australians have a lower life expectancy with increased risk of medical illness and dental disease compared to their non-Indigenous counterparts and their quality of life is much lower. ${ }^{7,8}$ Therefore these communities have a unique social profile, which requires specific approaches in the delivery of general and oral health care. It is well documented that European colonisation has brought about a complete change in the lifestyle of this group of people. ${ }^{7}$ Historically oral diseases were not reported among this group of people. However at present the rate of caries of Aboriginal children is twice the amount reported for non-Indigenous children. ${ }^{9}$ Although dental caries rates have remained low, tooth loss and periodontal disease have been reported as a significant problem in Indigenous adults. There are many reasons for the lack of good oral health in these communities all of which have their basis in cultural and historical background. Indigenous people are reluctant to attend mainstream oral health care services due to language barriers, and access to care due to geographic locations of residency and the long distances which they need to travel. Cultural barriers such as mainstream services not accommodating friends and family members when visiting the dentist has also been reported as a barrier to accessing services. ${ }^{10}$ This is especially significant since English is not the first language for some Indigenous people. Other reasons include the difficulty of retention of non-Indigenous oral health care workers in rural and remote areas of Australia, and the lack of appropriate cultural competency training of these workers. This situation is exacerbated by the unavailability of Aboriginal health care workers to support the mainstream services. In an attempt to alleviate issues of access to oral health care and prepare new graduates to work comfortably with Indigenous patients some dental schools have incorporated clinical training in an Indigenous context into their curricula in recent years. $^{11-13}$ 
This paper presents the development of a structured Indigenous dental outplacement programme by an Australian dental school and its evaluation based on students' feedback during 2008-2014. This is one of the few dental programmes specifically designed for the Indigenous community. This programme stands out on account of its offerings to all dental students (mandatory clinical placement) instead of a selected few in the cohort; and its successful mutual cooperation and sustained partnership with an Indigenous health care organisation since 2007. The other distinctive fact is that outplacement learning is assessed as part of the final year overall assessment (formative and summative). The educational philosophy as described by Biggs and Tang ${ }^{14}$ in regard to alignment of intended learning outcomes, learning tasks and the assessments have been followed during development of this programme and is described under Designing the curriculum for learning and service delivery. This programme is important from a national perspective since it would assist in training culturally informed oral health clinicians. This case report highlights the mutual benefits of outplacement programmes to students and Indigenous community. The development of the programme has been presented with reference to guidelines published in the literature. ${ }^{15}$

\section{Design and Methods}

\section{Designing the curriculum for learning and service delivery}

Clinical outplacements (outreach training) have specific advantages. The evaluation of these outplacement programmes indicates that there are benefits to dental students, dental schools, and the local communities. ${ }^{16,17}$ From a students' point of view the learning experience is different to the clinical education that they receive at a university hospital. The benefits to students from this approach to learning include the opportunities to work in a real world situation in their pregraduation years, which inspires and motivates them to learn. It allows them to provide clinical services to a wide range of patients (including those from different cultural and social backgrounds), to experience a variety of clinical procedures, and learn to work as part of the team at the clinical centre (service learning). The local communities benefit through the services provided by the dental students. At present most dental schools globally provide clinical training to students at clinical outplacements, sometimes at a great distance away from the dental school, some of which are located in rural and underserviced areas. Outplacements are designed to provide students a broad range of clinical experience and opportunity to develop knowledge of the oral health disparities among different communities including rural and Indigenous as compared to metropolitan.

In 2007, the Melbourne Dental School (MDS) developed an outplacement programme, called Rural Dental Rotation (RDR), for all dental students of Doctor of Dental Surgery (DDS, graduate) and Bachelor of Oral Health (BOH, undergraduate) courses. ${ }^{18}$ It is designed to develop knowledge of oral health issues of rural and Indigenous communities and equip students with skills to manage oral health related problems specific to these communities. Currently, groups of 14-16 final year students spend 5 weeks at a rural location (Shepparton, Victoria, Australia) engaged in clinical practice at 3 public dental clinics in the region including at an Indigenous dental clinic called Rumbalara Aboriginal Oral Health Centre (RAOHC). The MDS in partnership with Rumbalara Aboriginal Cooperation (RAC), Mooroopna (4 km south-west of Shepparton), developed the Indigenous Oral Health Outplacement where dental students engage in learning activities related to provision of oral health care to the local Indigenous community. Thus the Indigenous oral health clinical experience is an integral part of the final year of the DDS (formerly BDSc) and BOH programmes.

Specific learning related to Indigenous oral health issues in the DDS programme has been defined in terms of its graduate attributes, in the following statements: On graduation, DDS graduates will have developed: knowledge of the determinants of a 'healthy society' and the economic, political, psychological, social and cultural factors that contribute to the development and persistence of oral health and illness; knowledge of the health of Indigenous Australians including their history and cultural development and the ongoing oral health disparities of Indigenous people.

The BOH graduate attributes include: Attuned to cultural diversity value different cultures, be well-informed citizens able to contribute to their communities wherever they choose to live and work, have an understanding of the social and cultural diversity in our community, respect Indigenous knowledge, cultures and values.

To achieve these learning outcomes, appropriate Indigenous learning contents have been incorporated from the early years of the DDS and $\mathrm{BOH}$ programmes (years 1 and 2 respectively). At that stage students develop knowledge about Indigenous Australians in relation to their oral health status including the disparity that still exists when compared with the rest of the Australian population and issues related to access to care and social determinants of health. For example, DDS students engage in two 1 hour lectures in the first year semester one subject Introduction to Professional Dental Practice on Cultural Competence and Indigenous Oral Health. Students are provided with further learning resources related to Indigenous oral health and cultures, as self-directed learning through the university Learning Management System. Students participate in an initial cultural competency seminar of 2 hours in preparation for their clinical outplacement at RAOHC. This workshop is organised at the end of the $3^{\text {rd }}$ and $2^{\text {nd }}$ year of the DDS and BOH programmes respectively. The histories, the culture and the beliefs of Indigenous people and how these impact on their oral health and their attitude towards accessing oral health care services are discussed with the assistance of an Aboriginal healthcare worker. It is also aimed at providing essential information to assist students to feel comfortable working in the environment at the RAOHC during their outplacement. The workshop content is designed to stimulate the students' curiosity as it relates to Indigenous culture, oral health issues and barriers in relation to accessing care. This early didactic learning is followed by experiential learning at the rural Indigenous clinic during the final year.

The RAOHC is a community controlled health service located in a sizeable rural area (Greater Shepparton) which has 2082 Indigenous people equivalent to $3.4 \%$ of the population of the state of Victoria in Australia. RAOHC offers a wide range of culturally appropriate complete health care services including health promotion as well as emotional and spiritual wellbeing services to Aboriginal and Torres Strait Islander people in the Greater Shepparton Region (http://www.rumbalara.org.au/). The Indigenous people feel comfortable seeking health care in such a community managed environment. On the first day of their rural clinical outplacement each group of students participates in an orientation session at RAOHC conducted by the dental clinic staff. Students participate in clinical orientation and a cultural information session (2-3 hours) with an Elder of the community. This includes the history of the Aboriginal people in Shepparton, the specific history of Rumbalara including a tour of the premises and visit to a typical house to which Aboriginal people were allocated during the 1950s. The stories of the Aboriginal people are told by the Elder who engages the students in conversation, thus enhancing their cultural knowledge. During the clinical outplacement at RAOHC, students develop clinical skills to provide oral health care tailored to the community and experience aspects of Indigenous culture. They also have the opportunity to develop knowledge regarding issues related to access to oral health care through dialogue with the clients of RAOHC during their outplacement at 
Rumbalara. It is expected that educating dental students with awareness of cultural perspectives will better prepare them to work with the Indigenous people on graduation. ${ }^{10}$ ROAHC has provided MDS with 1-2 dental chairs and 2 students are rostered to the clinic per day. Each student experiences 2-3 days of clinical practice at RAOHC. The clinical load of the supervisor is reduced to allow effective clinical supervision of 2 dental students.

There are documented advantages of experiential learning. During their Indigenous clinical outplacement students go through the cycle of active experimentation and reflection. ${ }^{19}$ They learn through performing tasks or actions and through reflective observations. These observations and reflections provide new information to understand the experience which enables students to assess and solve problems. The role of clinical supervisors is vitally important in this process of experiential leaning where dental students build on the previously received didactic information ${ }^{20}$ and develop respect and appreciation for Indigenous culture and enhance their skills for service provision to Indigenous communities in the future.

Students' learning is assessed through reflective clinical logs which are required to be completed for every patient managed by the students. Students document the management of each patient including the treatments provided. They perform self-assessment using a letter grade and the criteria are as follows: $\mathrm{N}=$ no advice or assistance required for procedure; $M=$ minimal advice or assistance required for procedure; $\mathrm{A}$ = advice or assistance required for less than half of the procedure, and/or lack of organization; $U=$ advice or assistance required for more than half the procedure, and/or poor organization. The clinical supervisor also grades the student using these criteria. Assessment guidelines for students and clinical supervisors are as follows: Did the treatment proceed as planned? Were there any unforeseen problems? What did the student learn (improvement in skills, new information)? Did the student demonstrate appropriate level of knowledge required for the procedure? Students are required to document a reflective analysis of their performance, (analysis of events) and what actions they would take to improve management of their patients. Clinical supervisor feedback is provided during the clinical session and can be described as brief and/or formal feedback. ${ }^{21}$ Discussions include how students performed and how they can improve particular procedures.

Students are encouraged to observe, reflect and understand the overall experience in order to learn and complete the experiential learning cycle. The Indigenous dental assistants play a key role in guiding students during gathering information from patients regarding their reason for presenting, and collecting information on their medical, dental and social histories. Most adult patients visiting RAOHC are poly-morbid and thus treatment planning becomes more complex. Students experience cultural aspects. For example, Indigenous people visit the dentist accompanied by family and friends, and this culture has to be accommodated in the provision of oral health care. At the end of the clinical outplacement, students are assessed (group presentation, summative assessment) for their knowledge and understanding of Indigenous history, and the general and oral health issues of Indigenous community. This assessment is conducted as a written report of 2000 words submitted on the $4^{\text {th }}$ week of the rotation and a group oral presentation (60 minutes presentation and question and answer session of 20-30 minutes) on the last day of the rotation, at the wrap-up session. This assessment component is aligned with the learning outcomes for DDS and BOH students and is weighted $10 \%$ of the overall final year DDS assessment. The clinical logs (formative assessments) are also designed to align with the learning outcomes.

\section{Students' feedback}

On completion of each rotation students responded to a voluntary anonymous survey. This survey was conducted using a questionnaire to evaluate the effectiveness of the program using students' feedback. ${ }^{22}$ Questions were designed to obtain feedback on their overall experiences and learning achievements during RDR. Ethics approval was obtained for this survey from the Human Ethics Advisory Group of the University of Melbourne (Ethics approval: 0718660.1). To ensure confidentiality, all questionnaires were entered into database by an independent MDS staff member before any analysis was undertaken. The solicited responses to formal structured questions were on a 5-point Likert scale. Arithmetic mean, standard deviation and median were calculated to interpret and qualify the overall response against individual questions. A total of 746 dental students participated in the programme during 2007-2014 (Table 1). Feedback from students was collected from 2008 onwards (Table 2).

Five structured questions pertaining to RAOHC outplacement were analysed (Table 2). Questions 4 and 5 refer to knowledge and attitudes respectively. Response rate for these questions was about $89 \%$. Students agreed that the RAOHC outplacement increased their understanding of Indigenous issues which is a good outcome of this programme. The student feedback indicates the benefits of the programme to the students. All students met the requirement to pass the group assessment conducted at the wrap-up sessions and thus meeting the learning outcomes set out for this programme.

The year-wise students' feedback for the key question (4) regarding their learning had the average scores varying between 3.9-4.2 (from an

Table 1. Number of students attending Indigenous dental clinic (Rumbalara Aboriginal Oral Health Centre) in 2007-2014.

\begin{tabular}{lcccccccccc} 
Year & 2007 & 2008 & 2009 & 2010 & 2011 & 2012 & 2013 & 2014 & Total \\
Students* & 86 & 82 & 89 & 92 & 98 & 88 & 107 & 104 & 746 \\
\hline
\end{tabular}

*Includes students of BDSc (2008-13), DDS (2014), and BOH (2007-14).

Table 2. Students' feedback after the completion of Indigenous dental clinical outplacement at Rumbalara, 2008-2014.

\begin{tabular}{|c|c|c|c|c|c|c|c|c|c|c|c|}
\hline \multirow[t]{2}{*}{ Statements that students re } & \multicolumn{5}{|c|}{ Year-wise average score of students } & \multicolumn{2}{|c|}{ response* } & \multicolumn{4}{|c|}{ All years summary statistics* } \\
\hline & 2008 & 2009 & 2010 & 2011 & 2012 & 2013 & 2014 & Mean & Median & SD & Total \\
\hline 1) Induction at Rumbalara Clinic was useful & 4.0 & 4.0 & 3.7 & 3.6 & 4.0 & 4.1 & 3.6 & 3.86 & 4 & 0.86 & 604 \\
\hline 2) Clinical program at Rumbalara was well organized & 3.9 & 3.7 & 3.4 & 3.5 & 3.7 & 4.0 & 3.2 & 3.62 & 4 & 1.05 & 597 \\
\hline 3) Demonstrators and clinic staff were helpful & 4.6 & 4.5 & 4.2 & 4.3 & 4.3 & 4.4 & 3.7 & 4.28 & 4 & 0.83 & 596 \\
\hline 4) Improved my understanding of Indigenous issues & 4.2 & 4.3 & 3.9 & 4.0 & 4.2 & 4.2 & 4.0 & 4.10 & 4 & 0.85 & 630 \\
\hline 5) Increased the possibility that I will practice in Indigenous health & 3.7 & 3.6 & 3.4 & 3.7 & 3.7 & 4.0 & 3.6 & 3.66 & 4 & 1.04 & 627 \\
\hline Year-wise responses (n) & 72 & 87 & 89 & 96 & 86 & 106 & 104 & & & & \\
\hline
\end{tabular}

*Likert scale: 1. Strongly disagree; 2. Disagree; 3. Undecided; 4. Agree; 5. Strongly agree 
overall score of 5 , where 5 refers to strongly agree) over the six-year period analysed (Table 2). It is interesting to note that on average students are more positive about the likelihood of working in Indigenous oral health (Score 3.4-4.0; Question 5). All the scores show a slight decline in 2010 , which may be attributed to the changes in clinical staff and disruptions of services due to refurbishment of the clinic in that year.

The analysis of cumulative responses of students from 2008 till 2014 was also carried out (Table 2). Students found the Indigenous outplacement programme well organised (mean \pm SD: $3.62 \pm 0.86$ ). Students agreed that this programme improved their understanding of Indigenous issues $(4.10 \pm 0.8)$ and increased the possibility that they will practice in Indigenous health $(3.66 \pm 1.0)$. They were pleased with the assistance received by clinical supervisors and clinic staff at RAOHC $(4.28 \pm 0.8)$.

The open ended questions have not been analysed in this study. However some selected comments have been presented here as examples: The clinical staff has a good knowledge of the community and were able to provide us with important cultural information of the patients presenting to the clinic. This assisted us in managing patients to get good oral health outcomes. The orientation made me more aware about Aboriginal culture. Cultural sensitivity induction at Rumbalara was informative and adequate. Uncle (the Elder) did a good job, was interesting and helpful. It prepared us well for the rotation at Rumbalara.

\section{Discussion}

This study has provided an overview of the essential components of an Indigenous dental outplacement programme in Australian context. It has been demonstrated how the development, implementation and evaluation of such a programme can be achieved for the oral health benefits of an Indigenous community.

It is to be noted that the results presented in this paper are mainly based on the feedback from students. Feedback from community and staff members will form part of future studies.

In the words of Bainbridge et al. ${ }^{10}$ Cultural competence is more than cultural awareness - it is the set of behaviours, attitudes, and policies that come together to enable a system, agency, or professionals to work effectively in cross-cultural situations. Indigenous dental outplacement programmes provide a cross-cultural setting where students have the opportunity to develop the appropriate attitudes and behaviours. It provides experiential learning within an oral health care system where students apply their didactic knowledge into clinical practice and learn to work as a professional team. It increases opportunities of interdisciplinary partnerships among health professionals and has the potential to improve the delivery of health care in underserviced areas as demonstrated by a programme in North America. ${ }^{23}$

The Indigenous oral health outplacement of the MDS has successfully provided oral health services at the public dental clinic at Rumbalara since 2007 while providing learning experience to students. Students' logs indicate they have managed on average 2-3 patients per 3.5 hour clinical session and logged reflective comments which indicate they have met their learning outcomes. Similar studies have shown that inclusion of specific learning and teaching of Indigenous oral health modules into dental educational programmes assist in fostering culturally informed clinician-patient relationships. ${ }^{11,13,24}$
The contribution of Indigenous health care workers including dental assistants in the training of graduates has to be acknowledged and they need to be encouraged and supported to continue to participate in these activities.

The programme allowed graduates to participate in the provision of much needed oral health care to the Australian Indigenous population in the region. However there have been some shortcomings with the programme in recent years due to the high turnover rate of clinical supervisors (shown via feedback of 2014, Table 2). As a result, students found it difficult to receive consistent feedback from clinical supervisors. MDS staff has provided support and advice during recruitment of clinical supervisors and currently an experienced senior dentist has taken responsibility of overseeing the student programme at RAOHC. MDS continues to provide support to the practice manager at RAOHC, and assist with education of the clinical supervisors to support student learning. In some instances the rural and Indigenous dental clinics are under-resourced as Lalloo et al. ${ }^{11}$ reported the constant breakdown of equipment at one Indigenous outplacement. In the present study, however, the students were pleased to work in the new clinical facilities at RAOHC especially since they are well equipped rooms unlike the open plan clinics at the main metropolitan hospital. Similar to other reports, ${ }^{11}$ non-attendance of Indigenous patients has been observed in this study. The non-attendance of patients is recorded in the students' clinical logs.

Overall feedback from students and the partner organisation (personal communications) has been positive. This is mainly due to the positive outcomes achieved by students in oral health promotion and the provision of dental services to the community. The academic team at MDS has contributed in many ways to support the staff at Rumbalara and have remained engaged with the administration. It is hoped that the exposure to an environment such as RAOHC would encourage graduates to participate in provision of oral health services to Indigenous Australians upon graduation. Currently one graduate from MDS is employed as a part time clinician at the RAOHC indicating the positive results of the programme. The patients' informal feedback of the programme is positive and the Indigenous community appreciates the services provided by the students. MDS has planned to evaluate the programme through patients' experience of student services including the types and extent of oral health services provided by students in the future. Improved cultural awareness amongst graduates would allow them to work more effectively with Australian Indigenous communities. Future research on graduate work patterns, assessment of the potential gains (health outcomes) by the Indigenous community and review of the cost effectiveness of the programme is required to fully evaluate the impact of this programme. ${ }^{25}$

\section{Conclusions}

The case study of an Indigenous clinical outplacement programme, sustained over six years, has demonstrated that mutual benefits can be achieved through student participation in the provision of services in the public health sector. The programme established by the Melbourne Dental School has shown beneficial learning outcomes for students. It provides students an opportunity to learn cultural safety and address oral health needs of the local Indigenous community. 
Correspondence: Menaka Arundathi Abuzar, Melbourne Dental School, University of Melbourne, Victoria 3010, Australia.

Tel.: +61.9341.1495.

E-mail: maabuzar@unimelb.edu.au

Key words: Indigenous community; oral health; dental students; clinical outplacement.

Acknowledgements: the continued support of the staff at Rumbalara Aboriginal Oral Health Centre (RAOHC) for the outplacement programme is greatly appreciated.

Contributions: MAA has taken a key leadership role in the development of this programme and has contributed in the preparation of this manuscript; JO has contributed with the management of the programme, and has contributed to this manuscript.

Conflict of interest: the authors declare no potential conflict of interest.

Received for publication: 15 December 2015.

Accepted for publication: 14 March 2016.

(C) Copyright MA. Abuzar and J. Owen, 2016

Licensee PAGEPress, Italy

Journal of Public Health Research 2016;5:668

doi:10.4081/jphr.2016.668

This work is licensed under a Creative Commons Attribution NonCommercial 4.0 License (CC BY-NC 4.0).

\section{References}

1. Gracey M, King M. Indigenous health part 1: determinants and disease patterns. The Lancet 2009;374:65-75.

2. Mejia GC, Parker EJ, Jamieson LM. An introduction to oral health inequalities among Indigenous and non-Indigenous populations. Int Dent J 2010;60:212-5.

3. Patel J, Hearn L, Gibson B, Slack-Smith LM. International approaches to indigenous dental care: what can we learn? Aust Dent J 2014;59:439-45.

4. WHO. Health of Indigenous people. Fact Sheet No 326. Geneva: World Health Organisation; 2007.

5. UN. Indigenous people, Indigenous voices: who are indigenous people? Fifth Session of UNPFII, Fact Sheet 1. New York: UN; 2006.

6. AIHW. The indigenous population. Canberra: Australian Institute of Health and Welfare; 2015.

7. Williams S, Jamieson L, MacRae A, Gray C. Review of indigenous oral health. 2011. Available from: http://www.healthinfonet. ecu.edu.au/uploads/docs/oral_health_review_2011.pdf

8. AIHW. The health and welfare of Australia's Aboriginal and Torres Strait Islander people: an overview. Canberra: Australian Institute of Health and Welfare; 2011.

9. Roberts-Thomson K, Australian Research Centre for Population
Oral Health, The University of Adelaide, South Australia. Oral health of Aboriginal Australians. Austral Dent J 2004;49:151-3.

10. Bainbridge R, McCalman J, Clifford A, Tsey K. Cultural competency in the delivery of health services for Indigenous people. Issues paper no 13 produced for the Closing the Gap Clearinghouse. Canberra: Australian Institue of Health and Welfare, Australian Government; 2015.

11. Lalloo R, Evans JL, Johnson NW. Dental students' reflections on clinical placement in a rural and indigenous community in Australia. J Dent Educ 2013;77:1193-201.

12. Bazen J, Paul D, Tennant M. An aboriginal and torres strait islander oral health curriculum framework: development experiences in Western Australia. Aust Dent J 2007;52:86-92.

13. Bazen JJ, Kruger E, Dyson K, Tennant M. An innovation in Australian dental education: rural, remote and indigenous pregraduation placements. Rural Remote Health 2007;7:703.

14. Biggs J, Tang, C. Teaching for quality learning at university: what the student does. 3rd ed. Berkshire: McGraw Hill; 2007.

15. Reznich CB, Anderson WA. A suggested outline for writing curriculum development journal articles: the IDCRD format. Teach Learn Med 2001;13:4-8.

16. Smith M, Ash P, Gilmour ASM, et al. Outreach training: the special interest group's report. Eur J Dent Educ 2011;15:85-9.

17. Eaton KA, De Vries J, Widström E, et al. Schools without walls? Developments and challenges in dental outreach teaching - report of a recent symposium. Eur J Dent Educ 2006;10:186-91.

18. Abuzar MA, Burrow MF, Morgan M. Development of a rural outplacement programme for dental undergraduates: students' perceptions. Eur J Dent Educ 2009;13:233-9.

19. Kolb DA. Experiential learning: experience as a source of learning and development. Englewood Cliff: Prentice Hall; 1984.

20. Mullins G, Wetheral, J, Robbe, I. Learning in the clinical environment. In: Sweet J, Huttly, S, Taylor, I, eds. Effective Learning and teaching in medical, dental and veterinary education. London: Kogan Page Ltd; 2003. pp 169-179.

21. Branch WTJ, Paranjape A. Feedback and reflection: teaching methods for clinical settings. Acad Med 2002;77:1185-8.

22. Belfield C, Thomas H, Bullock A, et al. Measuring effectiveness for best evidence medical education: a discussion. Med Teach 2001;23:164-70.

23. Ballweg R, Berg J, DeRouen T, et al. Expanding dental education partnerships beyond the four walls. J Dent Educ 2011;75:300-9.

24. Bulgarelli A, Roperto R, Mestriner S, Mestriner W. Dentistry students' perceptions about an extramural experience with a Brazilian indigenous community. Indian J Dent Res 2012;23:498500.

25. Clifford A, McCalman J, Bainbridge R, Tsey K. Interventions to improve cultural competency in health care for Indigenous peoples of Australia, New Zealand, Canada and the USA: a systematic review. Int J Qual Health Care 2015;27:89-98. 